Research Article

\title{
Graph Theory-Based Mathematical Calculation Modeling for Temperature Distribution of LED Lights' Convective Cooled Heat Sinks under Moisture Environment
}

\author{
Bei-xuan Lyu (iD, ${ }^{1}$ Yu-ren Chen, ${ }^{2}$ and Yong $\mathrm{Li}^{1}$ \\ ${ }^{1}$ Department of Mechanical and Electrical Engineering, Hulunbeier Vocational Technical College, Hulunbeier, China \\ ${ }^{2}$ Department of Electronic Information Materials, School of Materials Science and Engineering, Shanghai University, \\ Shanghai, China
}

Correspondence should be addressed to Bei-xuan Lyu; shurobots@126.com

Received 11 December 2019; Revised 12 March 2020; Accepted 6 April 2020; Published 27 April 2020

Guest Editor: Sanghyuk Lee

Copyright $(2020$ Bei-xuan Lyu et al. This is an open access article distributed under the Creative Commons Attribution License, which permits unrestricted use, distribution, and reproduction in any medium, provided the original work is properly cited.

In this paper, a mathematical model based on graph theory is proposed to calculate the heat distribution of LED lights' convective cooled heat sink. First, the heat and mass transfer process of a single fin under moisture environment is analyzed. Then, the heat transfer process is characterized by a digraph, defining fins and joints of a heat sink as edges and vertices in graph theory. Finally, the whole heat transfer process is described by two criteria achieved based on graph theory. Therefore, the temperature-heat calculation equations of the whole heat sink are deduced. The accuracy of this model is verified by testing the junction temperature of different LED chips mounted on the same heat sink under moisture environment, and the relative errors between the calculated value and the experimental data are all within 5\%, and it is also concluded from the model that heat sinks with an identical heat digraph but different types have close cooling performance and are verified by two typical heat sinks, cylindrical heat sink and rectangular plate-fin heat sink, under the same conditions. The mathematical model based on group theory developed in this paper combined with computer technology is convenient for the performance analysis among a large number of heat sink fin arrangement schemes.

\section{Introduction}

Nowadays, light-emitting diode (LED) has met an extensive application in every aspect of our life. According to some comprehensive studies' conclusions [1-3], high LED junction temperature will not reduce the service life of LEDs significantly yet has a negative impact on the optical performance, revealing the great significance of reasonable heat sink design to LED lanterns. Now, extensive research studies have been conducted well and widely, concerning heat sinks of LED lights to improve their cooling performance.

Mathematical models to analyze thermal characteristics of heat sinks are developed by various scholars in recent years, such as Sun [4], who established a model to predict the peak temperature of a LED bulb by analyzing the performance of its heat sink with effective heat conductivity, and according to simulations and experimental results in [4], Sun [5] extended the simplified mathematical model to estimate the heat flow of a LED bulb in the spherical coordinate system further and verified the validity of the model by numerical simulation with the explicit finite-difference method (EFDM), considering the actual working conditions. Jang et al. [6] developed a correlation model to predict the Nusselt number, which was validated experimentally, thus evaluating the influence of orientation effect on the heat dissipation capacity of an inclined cylindrical heat sink. Also, the model showed that the cooling performance escalated, accompanied with the increasing angle of inclination of the heat sink. Subsequently, they [7] supplemented the experiment to characterize the relationship between thermal resistance of a plate-fin heat sink and its installation angles on the basis of [6], which showed that the thermal resistance 
decreased first and then increased, according to the increased angle. Jang further detailed the analytical model in [8] to predict the thermal resistance of a plate-fin heat sink and correlated the installation angle with its essential design parameters at last. Lv and Xiong [9] deduced a temperature distribution model of a heat sink and verified its correctness by experiment and numerical simulation. Besides, they [10] established a mathematic model of LED heat sinks in greenhouses by the condition that the heat conducted into the fin heat equals the total heat from the air convection and the condensation of wet air.

Besides, many optimization methods were applied to balance the influence of various factors, e.g., mass, size, and number of fins to achieve optimal performance. Khan [11] optimized cylindrical pin-fin heat sinks by using the entropy generation minimization (EGM) method, concerning the influence of heat transfer and pressure drop. Ahmed [12] analyzed the influence of orientation effect on heat transfer performance of heat sinks and optimized heat sinks by numerical calculation and simulation. Lv and Liu maximized the capacity of a heat sink to dissipate heat by using a topology optimization method in [13]. By using the particle swarm optimization (PSO) algorithm, Lampio and Karvinen [14] optimized cooled heat sinks. Bar-Cohen [15] studied the total coefficient to measure the cooling capacity of heat sinks and optimized their performance by the least-energy method.

Lately, researchers also studied the cooling performance of heat sinks, which vary in different contractures, forms, and geometric parameters, by analyzing their thermal and flow characteristics, thus derived and innovated novel heat sinks. Schmid et al. [16] analyzed the influence of varying the inter-fin base length on the natural convective heat sinks by the numerical parametric study, and they found that interfin base length determined the optimal fin spacing after the comparison of heat sinks with two different fin orientations with the identical base area at the same condition. Yu et al. [17] found that it was impossible to meet the demand of achieving the optimal performance with a lightweight at the same time after performing parametric studies of a radial heat sink. Jeong et al. [18] proposed a modified plate-fin heat sink with openings and investigated the effects of its all design parameters on heat dissipation performance, and the novel structure can reduce $30.5 \%$ thermal resistance compared with the conventional no-opening form.

In a word, existing research mainly focuses on the study of novel heat sink development and optimization and improvement of heat sinks and the calculation model foundation in cooling performance. Graph theory is the study of graphs, which can analyze relationships among objects quantitatively and has a wide application in various fields, e.g., networking, algorithm, decision making, and electronics [19]. Especially in mechanism synthesis, a large number of mechanisms with similar performance can be automatically generated by using graph theory in computers [20]. Enlightened by this, the combination of graph theory and computer technology in order to evaluate the optimal design among a large number of heat sinks' fin arrangement swiftly will play a positive role in the development of LED cooling technology. In this study, based on our previous research work $[9,10]$ and the equivalent thermal admittance proposed in [21], we developed a mathematical model based on graph theory to evaluate the heat performance and thus laid the foundation for the follow-up development.

\section{Mathematical Model Based on Graph Theory}

2.1. Assumptions and Simplification. To carry out a steadystate analysis of a typical plate-fin exposed to moisture environment, as shown in Figure 1, the following essential assumptions, also known as the Murray-Gardner assumptions, are made to simplify this analysis:

(i) The fin material is isotropic, and its thermal conductivity remains constant in all directions

(ii) The thermal resistance of the condensed film is negligible

(iii) The latent heat of condensation of water vapor is unchanged

(iv) Compared with the heat flowing through the side of the fin, the heat flowing through the outermost part of the fin is neglected

(v) The influence of air pressure drops caused by airflow is neglected

(vi) The effect of heat radiation is ignored, and the heat sink surface is diffuse and gray

(vii) The flow is three dimensional and laminar

2.2. Fundamentals of Heat and Mass Transfer. A typical plate-fin and its terminology and coordinate system are shown in Figure 1. The origin of the length coordinate is set at the tip of the fin, and the positive sense is in a direction from the tip towards the base. In this process, mass transfer accompanied by the heat transfer and the heat conducted into the fin from LED chips equals the total energy taken away by the air convection and the condensation of wet air. Therefore, considering Fourier's law of heat conduction, Newton's law of cooling, and the law of mass transfer in wet air, these physical phenomena at any position $x$ along the length coordinate can be expressed in the following form:

$$
\begin{aligned}
& (q+d q)+2 \cdot(H+\delta) \cdot h \cdot\left(T-T_{a}\right) \cdot d x+2 \cdot(H+\delta) \\
& \cdot h_{D} \cdot i_{f g} \cdot\left(\omega_{a}-\omega\right) \cdot d x-q=0 .
\end{aligned}
$$

According to the Chilton-Colburn analogy [22], the relationship between heat transfer and mass transfer coefficients can be expressed by the following equation:

$$
\frac{h}{h_{D}}=c_{p} \cdot L e^{0.48}=c_{p} \cdot\left(\frac{\alpha}{D}\right)^{0.48} \text {. }
$$

Hence, if $\mathrm{H}>>\delta$ and the previous assumptions permit, the equation can be written in the following form:

$$
\frac{d^{2} \theta}{d x^{2}}-\left(\frac{2 \cdot h}{k \cdot \delta}\right) \cdot\left[\theta+\frac{i_{f g}}{c_{p} L e^{0.48}} \cdot\left(\omega-\omega_{a}\right)\right]=0,
$$




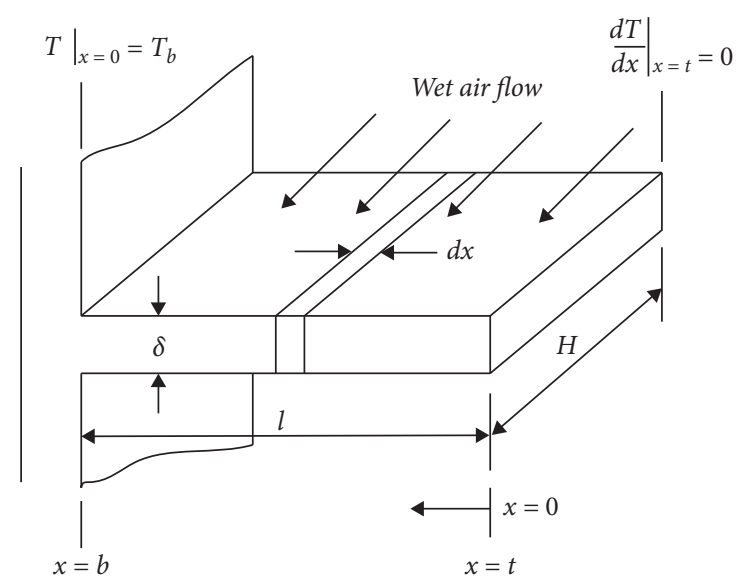

Figure 1: A fin of typical rectangular profile and its terminology and coordinate system.

where $\theta$ is the temperature excess between the fin and the surrounding environment. Considering the boundary conditions $\theta_{(x=b)}=\theta_{b}$ and $q_{(x=b)}=q_{b}$, we have

$$
\left[\begin{array}{ccc}
e^{m x} & e^{-m x} & -N \cdot\left(\omega-\omega_{a}\right) \\
k A m e^{m H} & -k A m e^{-m H} & 0
\end{array}\right] \cdot\left[\begin{array}{c}
C_{1} \\
C_{2} \\
1
\end{array}\right]=\left[\begin{array}{c}
\theta_{b} \\
q_{b}
\end{array}\right],
$$

where

$$
\begin{aligned}
& C_{1}=\frac{k \delta L m \cdot\left[\theta_{b}+N \cdot\left(\omega-\omega_{a}\right)\right] \cdot e^{-m H}+q_{b} \cdot e^{-m H}}{2 k \delta L m}, \\
& C_{2}=\frac{k \delta L m \cdot\left[\theta_{b}+N \cdot\left(\omega-\omega_{a}\right)\right] \cdot e^{m H}-q_{b} \cdot e^{m H}}{2 k \delta L m} .
\end{aligned}
$$

Equation (9) suggests the relationship between the temperature excess and the heat flow at position $\mathrm{x}$, and it can be rearranged as

$$
\begin{aligned}
& {\left[\begin{array}{l}
\theta_{t} \\
q_{t}
\end{array}\right]=} {\left[\begin{array}{cc}
\cosh (m H) & -\frac{1}{k \delta L m} \cdot \sinh (m H) \\
-k \delta L m \cdot \sinh (m H) & \cosh (m H)
\end{array}\right] \cdot\left[\begin{array}{l}
\theta_{b} \\
q_{b}
\end{array}\right] } \\
&+\left[\begin{array}{c}
{\left[\frac{1}{k \delta H m} \cdot \cosh (m l)-1\right] \cdot N\left(\omega-\omega_{a}\right)} \\
-\sinh (m l) \cdot N\left(\omega-\omega_{a}\right)
\end{array}\right] .
\end{aligned}
$$

\subsection{Mathematical Modeling Based on Graph Theory}

2.3.1. The Graph Model of Heat Flow Analysis. In graph theory, the graph is defined as a pair set $G=\left(V, E_{\psi}\right)$ and can satisfy the following conditions:

(1) $V$ is the nonempty set

(2) $E \subseteq[V]^{2}$; thus, the elements of $E$ are 2-element subsets of $V$
(3) Function $\psi: E \longrightarrow V \times V$

Here, $V$ denotes vertex-set and $E$ represents edge-set, and if all the elements of $E_{\psi}$ are ordered pairs, the graph is called digraph; if a graph in which the edges are not ordered, it is called an undirected graph. A graph $\left(V, E_{\psi}\right)$ with vertex-set $V=\left\{x_{1}, x_{2}, \ldots, x_{n}\right\}$ and edge-set $E=\left\{a_{1}, a_{2}, \ldots, a_{m}\right\}$ can be specified by the adjacency matrix, which is the $\mathrm{n} \times \mathrm{n}$ matrix A:

$$
A=\left(a_{i j}\right)_{n \times n}, \quad a_{i j}=\mu\left(x_{i}, x_{j}\right),
$$

where $\mu(x i, x j)$ is the number of edges connecting vertices $i$ and $\mathrm{j}$. Furthermore, a graph can also be represented by the $n \times m$ matrix $M$ called incidence matrix:

$$
M=\left(m_{x}(a)\right), \quad x \in V, a \in E .
$$

For the commonly used plate-fin heat sinks, they can be regarded as a composition of fins with joints arranged by different geometric requirements, and the joint is the junction connecting several fins, e.g., the $\mathrm{W}$-shaped and K-shaped heat sinks shown in Figure 2, respectively, are all composed of four fins, but with different numbers of joints. In order to simplify the procedure to get graph mappings from specific heat sinks, fins and joints of a heat sink as its elementary geometric components can be regarded as edges and vertices in graph theory, respectively. The undirected graph $G_{H}(V, E)$, in which the subscript $H$ represents the heat sink, characterizes the geometric characteristics of the heat sink, in which $V$ represents the joint set and $E$ represents the fin set, and its adjacency matrix $A_{H}$ of this undirected graph, which is the $n \times n$ matrix, can represent the actual geometric relationship of this heat sink.

When using the heat sink to transfer heat from LED chips to the surrounding environment, a heat transfer network is formed and can be characterized by a digraph $G_{F}$ $\left(V^{\prime}, E^{\prime}\right)$, in which subscript $F$ represents the heat flow, and this digraph is established on the basis of undirected graph $G_{H}$ by adding the following conditions:

(1) The joint around the heat source often is regarded as the start vertex of the digraph

(2) Adding an extra vertex as the end vertex in the digraph, considering the influence of the surrounding environment

(3) The edge-set $E^{\prime}$ is founded on the basis of making all edges in set $E$ of the graph $G_{H}(V, E)$ directional, and adding directed edges from all vertices except the start one towards end vertex

(4) Referring to the conservation of energy, constructing another directed edge starting from the end vertex, namely, representing the surrounding environment towards the start vertex, thus forming a closed loop of the digraph

If the undirected graph mapping geometric characteristics of the heat sink has these conditions $G_{H}(V, E),|V|=n$, and $|E|=m$, we can easily find that $\left|V^{\prime}\right|=n+1$ and $\left|E^{\prime}\right|=$ $m+n$ for the digraph $G_{F}\left(V^{\prime}, E^{\prime}\right)$, reflecting the actual heat exchange. Therefore, the heat transfer from LED chips to the 


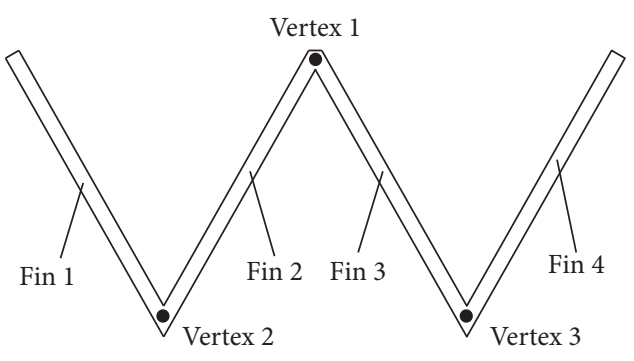

(a)

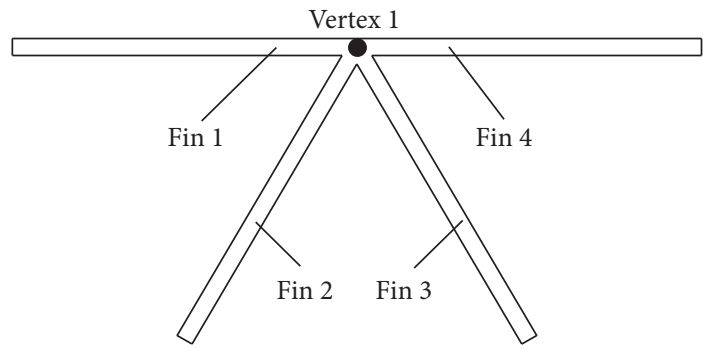

(b)

Figure 2: Two types of fin arrays with identical fin numbers but different joint numbers. (a) W-shaped fin array with four fins and three vertices. (b) K-shaped fin array with four fins and one vertex.

surrounding environment, inclusive of heat conduction and thermal convection, can be expressed by the digraph $G_{F}\left(V^{\prime}\right.$, $\left.E^{\prime}\right)$, namely, the association between multiple vertices and edges, and the whole heat flow can also be expressed by the incidence matrix $M_{F}$ by $(n+1) \times(m+n)$ and the elements $a_{i j}$ in $M_{F}$ are "1," "- 1 ," or " 0 " in accordance with the scheme:

$$
a_{i j}= \begin{cases}1, & \text { if the heat flow } j \text { leaves vertex } i, \\ -1, & \text { if the heat flow } j \text { enters vertex } i, \\ 0, & \text { if the heat flow } j \text { does not touch vertex } i .\end{cases}
$$

The heat generated by LED chips flows into the environment through several different fin branches; thus, there are multiple heat flow loops during the whole heat transfer process. In order to give an accurate expression, a matrix CF by $(n-1) \times(m+n)$ named as the heat flow loop matrix is introduced. The elements $c_{i j}$ in heat flow loop matrix $C_{F}$ are " 1 " or " 0 " in accordance with the scheme:

$$
c_{i j}= \begin{cases}1, & \text { if the heat flow } i \text { inclusive of edges } j, \\ 0, & \text { if the heat flow } i \text { exclusive of edges } j .\end{cases}
$$

Therefore, the geometric characteristics of a heat sink composed by $n$ vertices and $m$ edges, namely, $|V|=n,|E|=$ $m$, can be represented by an undirected graph $G_{H}(V, E)$, and the adjacency matrix $A_{H}$ is used to describe the geometric relationship of edges by $n$ vertices. After LED chips are mounted on the heat sink, a digraph $G_{F}\left(V^{\prime}, E^{\prime}\right)$ established by the undirected graph $G_{H}(V, E)$ is used to describe the heat generated by LED chips flowing towards the surrounding environment through the heat sink and for the digraph $G_{F}$ $\left(V^{\prime}, E^{\prime}\right)$, the incidence matrix MF by $(\mathrm{n}+1) \times(\mathrm{m}+\mathrm{n})$ and heat flow loop matrix CF by $(n-1) \times(m+n)$ are used to reflect the actual heat exchange. The specific calculation of LED temperature excess by matrix $M_{F}$ and $C_{F}$ is elaborated in the following section.

\subsubsection{Calculation of Thermal Admittance Matrix by Graph} Theory. The relationship between the temperature excess and the heat flow at both ends discussed in Section 2.2 can be analyzed by the application of the graph model to form the heat flow digraph. Therefore, after the linear transformation of equations (12), (13), and (14), the relation between heat flow and temperature excess may be written in the matrix form as

$$
\begin{aligned}
{\left[\begin{array}{l}
q_{b} \\
q_{a}
\end{array}\right]=} & {\left[\begin{array}{l}
k \delta L m \cdot \operatorname{coth}(\mathrm{mb})-k \delta L m \cdot \operatorname{csch}(\mathrm{mb}) \\
k \delta L m \cdot \operatorname{csch}(\mathrm{mb})-k \delta L m \cdot \operatorname{coth}(\mathrm{mb})
\end{array}\right] \cdot\left[\begin{array}{l}
\theta_{b} \\
\theta_{a}
\end{array}\right] } \\
+ & {\left[\begin{array}{c}
{\left[\frac{1}{k \delta H m} \cdot \cosh (\mathrm{ml})-1\right] \cdot N\left(\omega-\omega_{a}\right)} \\
-\sinh (\mathrm{ml}) \cdot N\left(\omega-\omega_{a}\right)
\end{array}\right], }
\end{aligned}
$$

and according to the definition of the equivalent thermal admittance $Y$, a parameter to measure heat transfer performance of the fin was proposed in [21], and its definition formula is as follows :

$$
Y=\frac{q}{\theta}=\frac{1}{R} .
$$

Figure 3 shows a typical thermal transmission case of a single flat-plate fin from the fin base to the fin tip, namely, from point $A$ to point $B$, representing thermal energy flows from high temperature to low temperature by heat conduction. As mentioned in Section 2.3.1, the heat flows from vertex $A$ towards vertex $B$, and the two vertices are all connecting the vertex $C$ which is representing the surrounding environment, thus forming two directional edges all towards the vertex $C$, but started from vertex $A$ and vertex $B$, respectively. We can give the following:

$$
\left\{\begin{array}{l}
Q_{A}=q_{1}+q_{2}, \\
Q_{B}=q_{1}-q_{3},
\end{array}\right.
$$

wherein heat flow $q_{1}$ and $q_{3}$ represent the heat quantity by convective heat transfer and $q_{2}$ represents the heat conducted through the fin. Referring to equation (13), each heat flow equals a product of admittance and a temperature excess:

$$
\left\{\begin{array}{l}
q_{1}=Y_{1}\left(\theta_{A}-\theta_{B}\right) \\
q_{2}=Y_{2} \theta_{A} \\
q_{3}=Y_{3} \theta_{B}
\end{array}\right.
$$




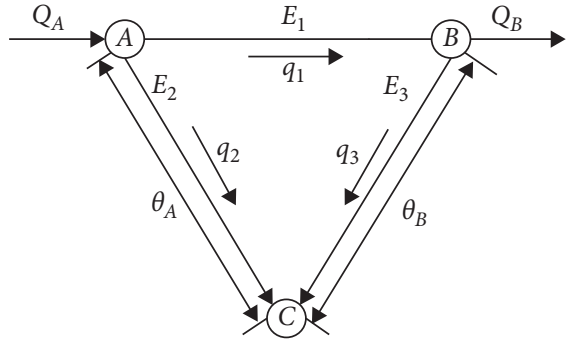

The surrounding environment

(a)

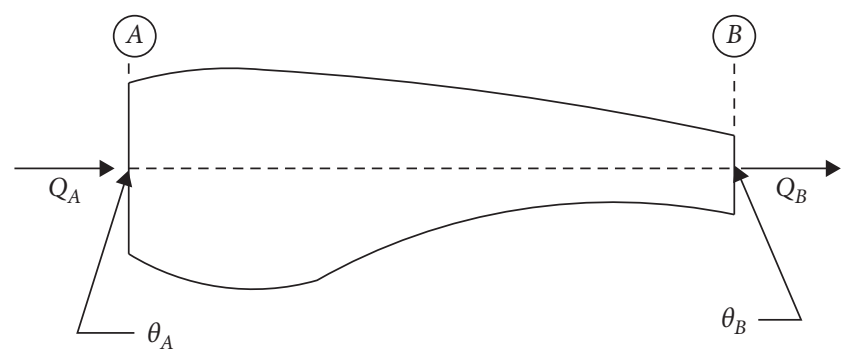

(b)

Figure 3: (a) The actual thermal transmission of a fin and its equivalent thermal digraph. (b) In the thermal digraph, the positive direction is set from high temperature towards low temperature.

wherein $Y_{2}$ and $Y_{3}$ represent the convection thermal admittance of edge 2 and edge 3 and $Y_{1}$ is measuring the conductive performance of edge 1. Considering the onedimensional Fourier equation, Newton's law of cooling, and the law of mass transfer in wet air, we have

$$
\left\{\begin{array}{l}
q_{2}=k \delta H \cdot \frac{\theta_{A}-\theta_{B}}{l}, \\
q_{1}=2 \cdot(H+\delta) \cdot\left[h \cdot \theta_{a}+h_{D} \cdot i_{f g} \cdot\left(\omega_{a}-\omega\right)\right], \\
q_{3}=2 \cdot(H+\delta) \cdot\left[h \cdot \theta_{b}+h_{D} \cdot i_{f g} \cdot\left(\omega_{a}-\omega\right)\right] .
\end{array}\right.
$$

Referring to equation (15), we obtain

$$
\left\{\begin{array}{l}
Y_{1}=\frac{k \delta H}{l} \\
Y_{2}=2 \cdot(H+\delta) \cdot h \\
Y_{3}=2 \cdot(H+\delta) \cdot h
\end{array}\right.
$$

As the basic geometric composite unit of the whole heat sink, each fin has limited capacity to transfer heat into the environment, and its undissipated heat is transferred to other fins by heat conduction. Therefore, heat will be spread on the whole heat sink by conductive transfer, namely, through the fins connected to form the heat sink, in order to exchange the heat into the environment by convective transfer. Obviously, heat conduction does not consume heat in this process, and according to the definitions mentioned above, the convective heat transferred into the environment by a heat sink with $n$ vertices can be calculated as follows:

$$
Q=\sum_{i=1}^{n-1} Y_{i} \cdot\left(T_{\text {LED }}-T_{\text {Ambient }}\right)
$$

wherein $Y_{i}$ represents the convective thermal admittance.

Referring to the conservation of energy and relevant contents mentioned above, the whole heat transfer process of the LED lights by using heat sinks can be described by the following criteria:

(i) The algebraic sum of the heat flow at each vertex in the graph is zero (ii) The algebraic sum of temperature difference of each edge along any flow in the graph is zero

They can also be expressed by the following equations:

$$
M_{F} \cdot \widetilde{q}=O
$$

and

$$
C_{F} \cdot \tilde{\theta}=O,
$$

wherein $\widetilde{\mathbf{q}}$ is the heat flux column vector by $(m+n) \times 1, \widetilde{\boldsymbol{\theta}}$ is the temperature excess column by $(n-1) \times 1$, respectively, and $O$ is the zero vector. By associating equations (18), (19), and (20), the temperature calculation equations of the whole heat sink is deduced. Therefore, the incidence matrix $M_{F}$ and the heat flow loop matrix $C_{F}$, all derived from matrix $A_{H}$, are the key to finish the temperature calculation.

2.3.3. Identical Digraph of Different Heat Sinks. In graph theory, isomorphic digraphs, as the two shown in Figure 4, have the exact same pattern, and the sole difference is the label of their vertices and edges. In general, if digraphs $G$ and $\mathrm{H}$ are isomorphic, it can be written $\mathrm{G} \cong \mathrm{H}$. Besides, there are bijections $\theta: V(D) \longrightarrow V(H)$ and $\varphi: E_{\psi}(D) \longrightarrow E_{\psi},(H)$, thus making $a \in E_{\psi}(D)$ have $\psi(a)=(x, y)$ and $\psi^{\prime}(\varphi(\mathrm{a}))=(\theta(x)$ and $\theta(y)) \in E_{\psi},(H)$; such a pair of mappings is called an isomorphism between $\mathrm{G}$ and $\mathrm{H}$.

Hence, based on the isomorphic digraphs concept, identical digraphs to characterize heat transfer from LED chips to the surrounding environment through heat sinks, even with different types, must satisfy the following conditions:

(1) The two digraphs are isomorphic, and they have the same digraph pattern and directions of each directed edges

(2) The thermal admittance of one edge in a digraph and the mapping edge in its digraph pair is equal

\section{Results and Discussion}

\subsection{Case 1: Verification under Moisture Environment}

3.1.1. Experimental Apparatus and Procedure. The test heat sink is made by grinding extruded aluminum alloy 6063 to 


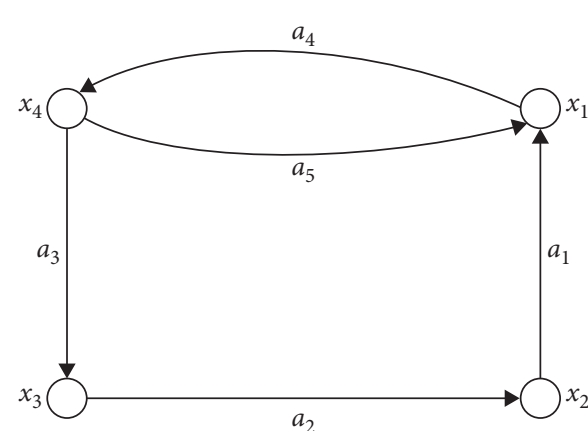

(a)

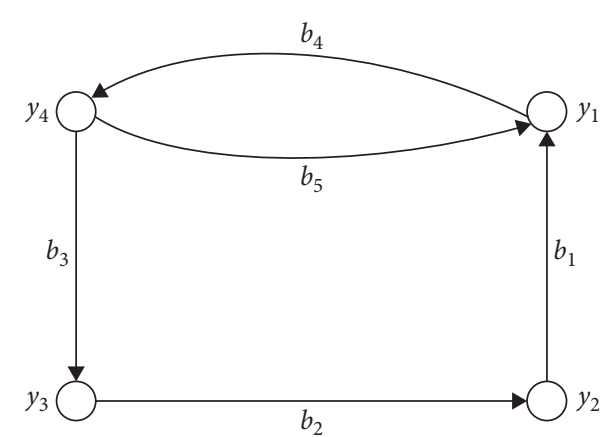

(b)

Figure 4: Two digraphs (a,b) represented by the same pattern.

$0.8 \mu \mathrm{m}$ roughness by $\mathrm{CNC}$ milling machine, thus reducing radiation emissivity, therefore, increasing the accuracy of the measurement. The main component's materials and thermal performance parameters are shown in Table 1 and the detailed dimensions are shown in Figure 5. In order to verify the mathematical model, the junction temperature of $20 \mathrm{~W}$, $25 \mathrm{~W}, 30 \mathrm{~W}, 35 \mathrm{~W}$, and $40 \mathrm{~W}$ LED chips mounted on the same heat sink was measured at 50\%-100\% relative humidity with T-type thermocouple and the specific experimental apparatus is shown in Figure 6. Therefore, we place the fins of the heat sink upright, namely, parallel to the gravity direction, to avoid the influence of the orientation effect. The temperature of the experimental environment is controlled at $20^{\circ} \mathrm{C}$, and the data acquisition started 1 hour after the LED lamp is lighted when the LED chip reaches a stable level.

3.1.2. Calculation Based on Graph Theory. The heat sink shown in Figure 5 presents a typical symmetrical structure; thus, we reduce computation by analyzing the heat flow of one side; therefore, the amount of heat generated by the LED chip is half. We achieve the digraph shown in Figure 7 (b) which is mapping the actual heat transfer through the heat sink. In this digraph, the vertex 1 marked in red circle is the heat source, also representing the start vertex in the digraph, and the vertices in blue circles represent the actual joints of this heat sink. Besides vertex 9, namely, the vertex in gray in Figure $7(\mathrm{~b})$ is the extra vertex acting as the end vertex mentioned in Section 2.2, thus considering the influence of the surrounding environment. Black lines in solid connecting vertices, which are mapping the actual geometrical structure of the heat sink, indicate the heat transfer by conduction through the heat sink pointed from high to low temperature, and black dash lines denote all vertices in blue circles which transfer heat from heat sink towards the environment by thermal convection. In addition, the red dash line, namely, the $E_{15}$ in Figure $7(\mathrm{~b})$ denotes an illusory heat flow from the environment towards the heat source, considering the conservation of energy and relevant contents mentioned in Section 2.3.2, thus forming closed loops for calculation.

Hence, the heat generated by LED chips, thus starts from the vertex 1, along seven flow loops, all inclusive of heat conduction and thermal convection, denoted by solid lines and dash lines, respectively, in Figure 7(b) to finish the whole thermal circulation, and the flow matrixes of the seven flow loops are as follows:

$$
\begin{aligned}
& C_{1}=\left\{e_{1}, e_{2}, e_{8}, e_{15}\right\}, \\
& C_{2}=\left\{e_{1}, e_{9}, e_{15}\right\}, \\
& C_{3}=\left\{e_{3}, e_{10}, e_{15}\right\}, \\
& C_{4}=\left\{e_{3}, e_{4}, e_{11}, e_{15}\right\}, \\
& C_{5}=\left\{e_{3}, e_{4}, e_{5}, e_{12}, e_{15}\right\}, \\
& C_{6}=\left\{e_{6}, e_{7}, e_{14}, e_{15}\right\}, \\
& C_{7}=\left\{e_{6}, e_{13}, e_{15}\right\},
\end{aligned}
$$

The matrix $A_{H}$ by $n \times n$ can be easily achieved. Besides, by analyzing the heat flow, we achieved the incidence matrix $M_{F}$ and the heat flow loop matrix $C_{F}$, and all the corresponding matrixes for calculation are shown in Table 2. Referring to equations (19) and (20), we got curves between relative humidity and junction temperature of $20 \mathrm{~W}, 25 \mathrm{~W}$, $30 \mathrm{~W}, 35 \mathrm{~W}$, and $40 \mathrm{~W}$ LED by calculation and conducted experiments for comparison.

3.1.3. Results and Analysis. Figure 8 shows the calculational and experimental relation curves between relative humidity and junction temperature of 20W, 25W, 30W, 35W, and 40W LED chips. All curves present the same change trend, the junction temperature of all kinds increase with the increasing ambient humidity, and the changing trend of calculation curves is basically consistent with that of experimental curves, namely, the junction temperature of LED increasing with the increase of humidity. Comparing the difference between calculational and experimental values, all the temperature differences between the measured and calculated values are all within $5^{\circ} \mathrm{C}$, namely, the relative errors between the calculated value and the experimental data are all within $5 \%$. Therefore, the correctness of the temperature distribution calculation model proposed in this paper is in good agreement with the experimental measurements. 
TABLE 1: Main component's materials and thermal performance parameters.

\begin{tabular}{lccccc}
\hline Components & Materials & Density $/ \mathrm{kg} \cdot \mathrm{m}^{-3}$ & Conductivity $/ \mathrm{W} \cdot \mathrm{m}^{-1} \mathrm{~K}^{-1}$ & Specific heat $/ \mathrm{J} \cdot \mathrm{kg}^{-1} \mathrm{~K}^{-1}$ & Thermal emissivity $^{-}$ \\
\hline Heat sink & Polished aluminum alloy 6063 & 2690 & 202 & 900 & 0.1 \\
LED chip & Semiconductor material & 6150 & 130 & 417 & - \\
\hline
\end{tabular}

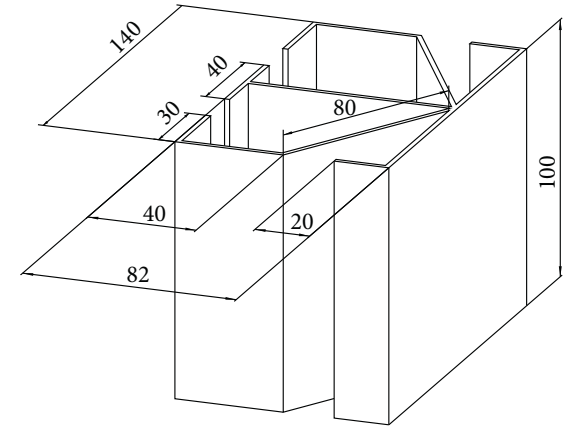

Figure 5: The heat sink and its specific dimensions.

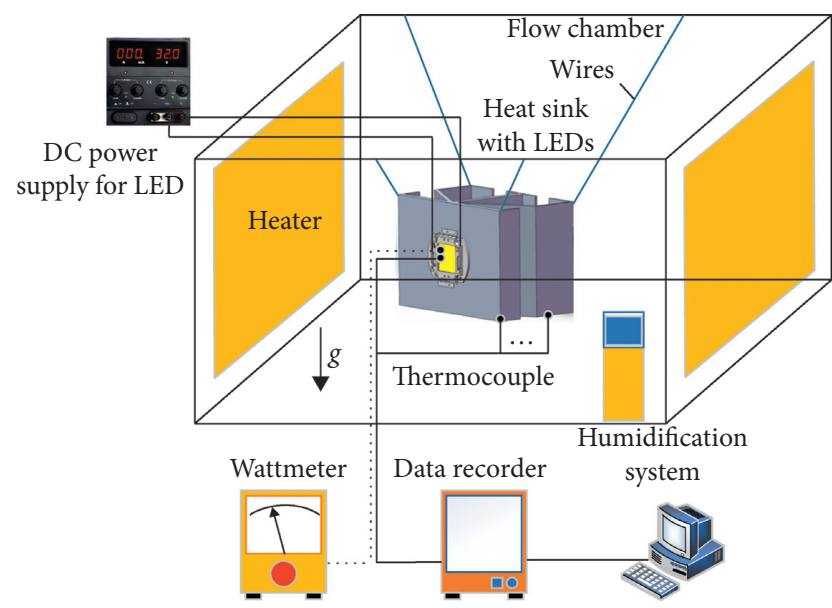

FIGURE 6: Simplified model of the experimental apparatus.

\subsection{Case 2: Verification of Close Cooling Performance of Heat} Sinks with Identical Heat Digraphs but Different Types. As shown in Figure 8, a cylindrical heat sink and a rectangular plate-fin heat sink are used in this case, and for the rectangular plate-fin heat sink, we treat its base as a joint such as the cylindrical one, thus making the two heat sinks sharing a similar heat flow digraph. Referring to equation (15) in Section 2.3.2, the equivalent thermal admittance $Y$ of each edge is equal, considering all fins of the two heat sinks having identical geometry size under the same working condition. Thus, it can be easily deduced from the model mentioned above that heat sinks sharing an identical heat digraph but different types have close cooling performance. In order to verify this conclusion, we tested the junction temperature of $20^{\circ} \mathrm{W}$ LED chips, respectively, mounted on two typical heat sinks, cylindrical heat sink and rectangular plate-fin heat sink, all with 6 fins of the same size, apparently belonging to two

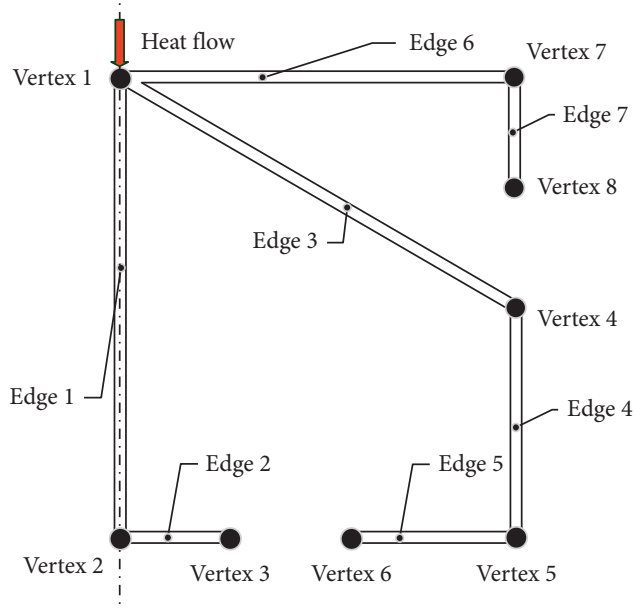

(a)

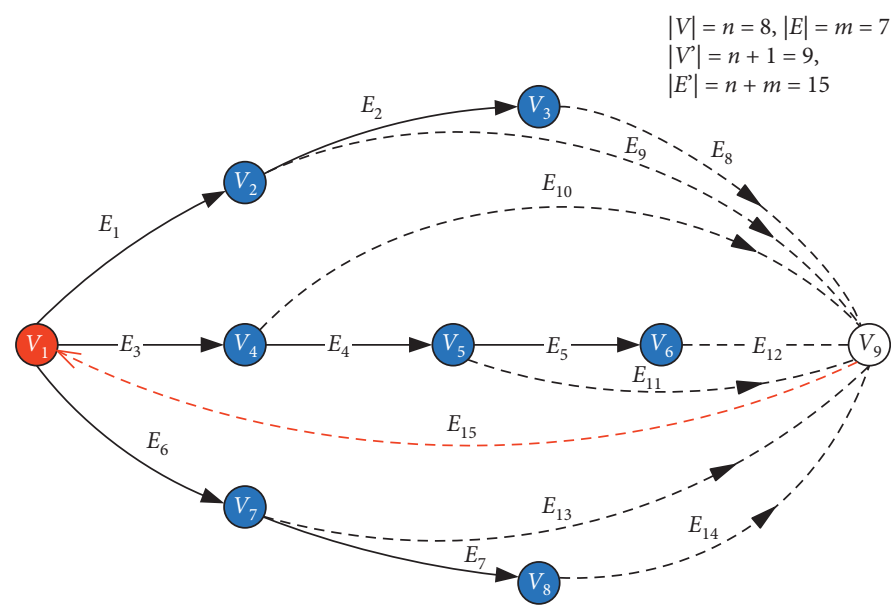

$\longrightarrow$ Heat flow by conduction

$\rightarrow$ Heat flow by convection

$--\rightarrow$ An illusory heat flow from the environment towards the LED

(b)

FIGURE 7: Vertices and edges of the heat sink (a) and the digraph of the heat flow (b).

different geometrical structures but sharing with an identical heat sink graph, as shown in Figures 8 and 9, respectively, using the same experimental method and heat sink materials, as mentioned in Section 3.1.

We achieve the digraph shown in Figure 10 which maps the actual heat transfer through the heat sink, the heat along with six flow loops expressed by equation (22), to finish the whole thermal circulation, and corresponding matrixes for calculation are shown in Table 3: 
TABLE 2: The matrix representation of the heat flow graph.

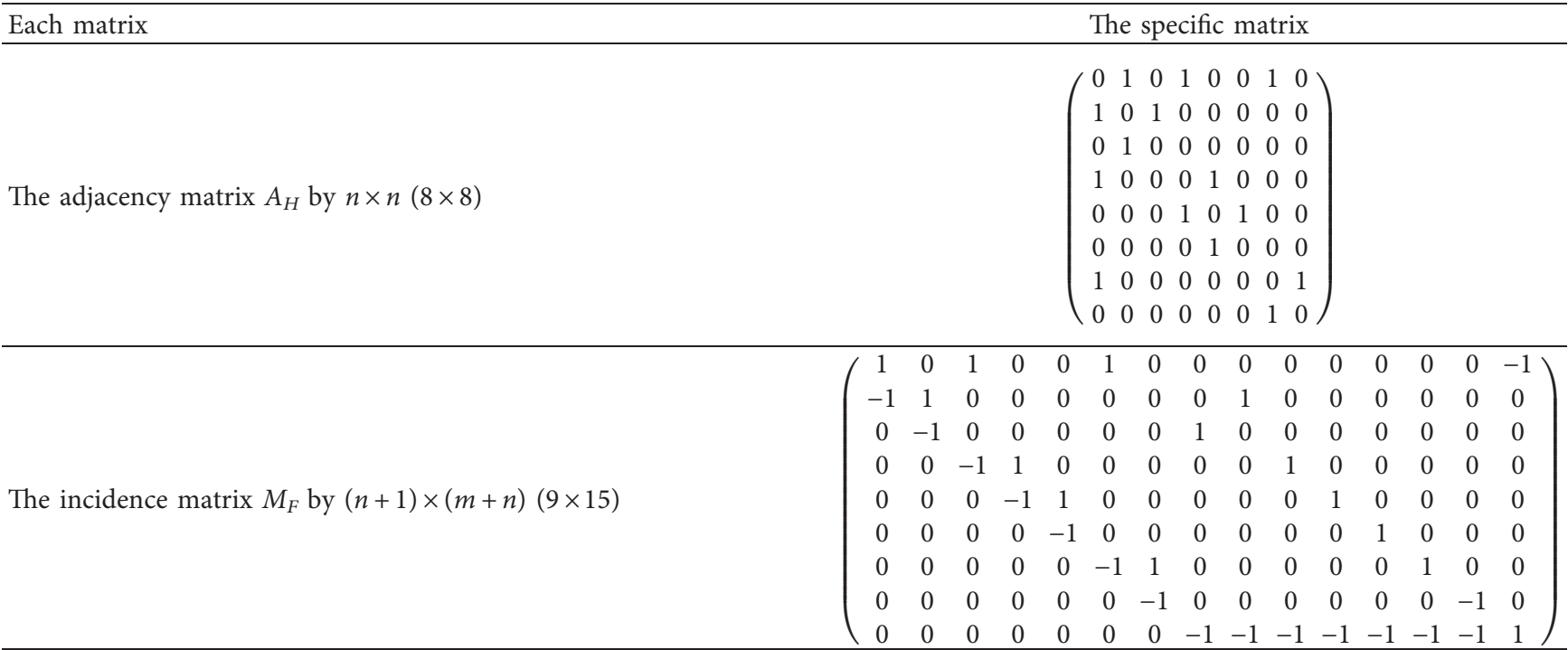

The flow matrix CF by $(n-1) \times(\mathrm{m}+\mathrm{n})(7 \times 15) \quad\left(\begin{array}{lllllllllllllll}1 & 1 & 0 & 0 & 0 & 0 & 0 & 1 & 0 & 0 & 0 & 0 & 0 & 0 & 1 \\ 1 & 0 & 0 & 0 & 0 & 0 & 0 & 0 & 1 & 0 & 0 & 0 & 0 & 0 & 1 \\ 0 & 0 & 1 & 0 & 0 & 0 & 0 & 0 & 0 & 1 & 0 & 0 & 0 & 0 & 1 \\ 0 & 0 & 1 & 1 & 0 & 0 & 0 & 0 & 0 & 0 & 1 & 0 & 0 & 0 & 1 \\ 0 & 0 & 1 & 1 & 1 & 0 & 0 & 0 & 0 & 0 & 0 & 1 & 0 & 0 & 1 \\ 0 & 0 & 0 & 0 & 0 & 1 & 1 & 0 & 0 & 0 & 0 & 0 & 0 & 1 & 1 \\ 0 & 0 & 0 & 0 & 0 & 1 & 0 & 0 & 0 & 0 & 0 & 0 & 1 & 0 & 1\end{array}\right)$

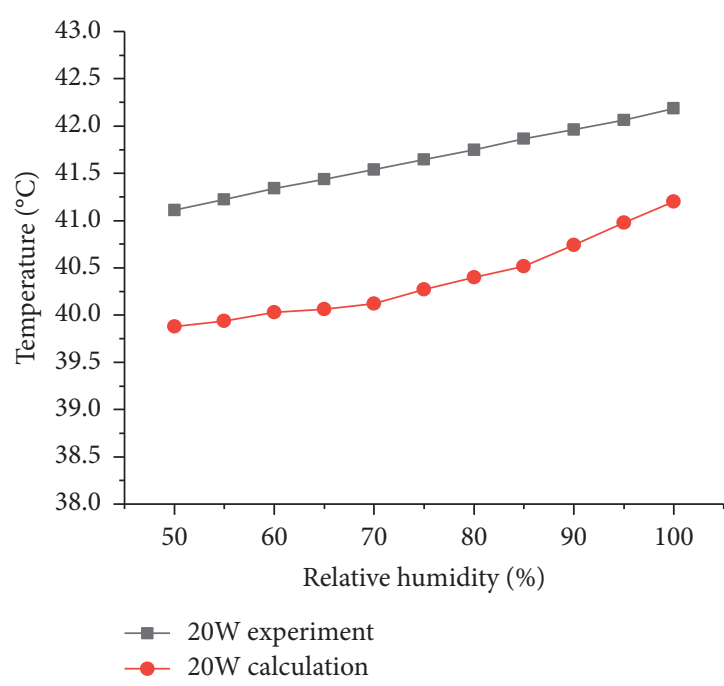

(a)

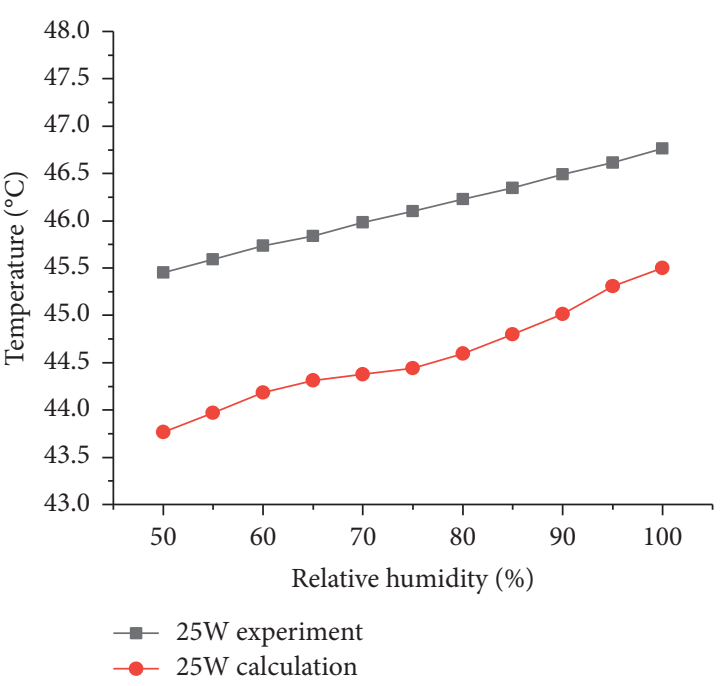

(b)

Figure 8: Continued. 

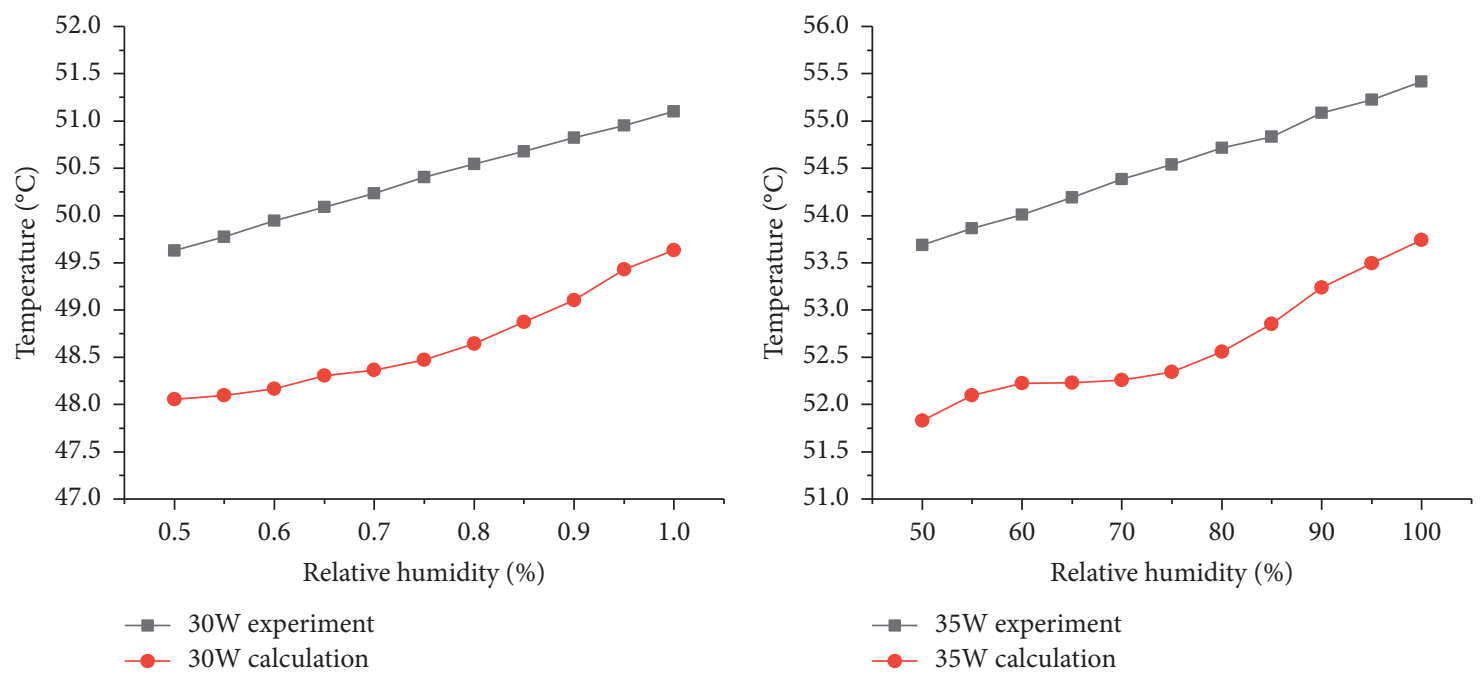

(c)

(d)

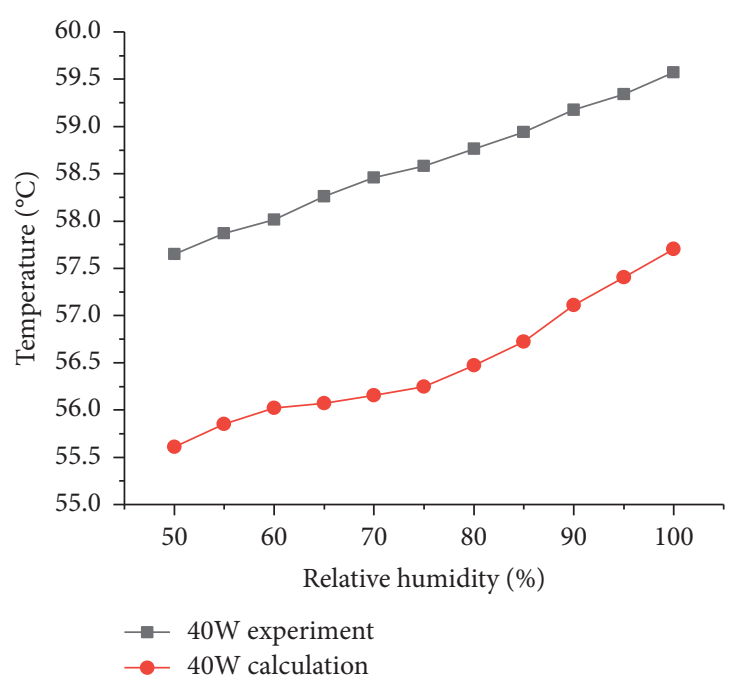

(e)

FIGURE 8: Calculational and experimental relation curves between relative humidity and junction temperature of 20W, 25W, 30W, 35W, and 40W LED chips.

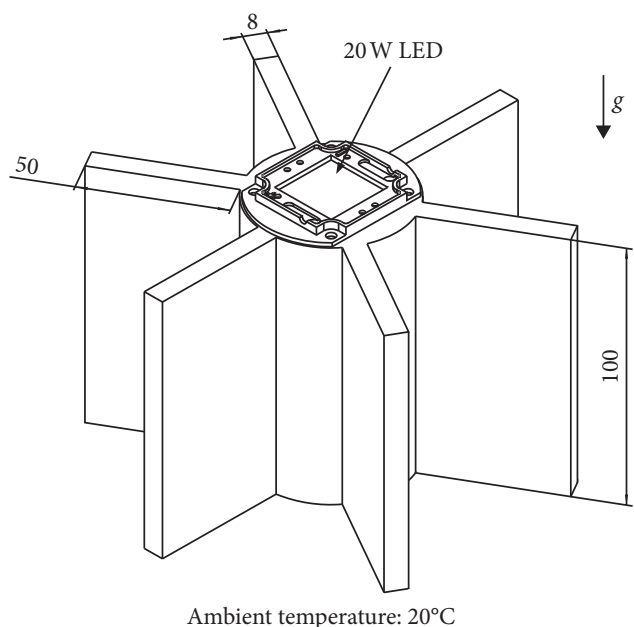

(a)

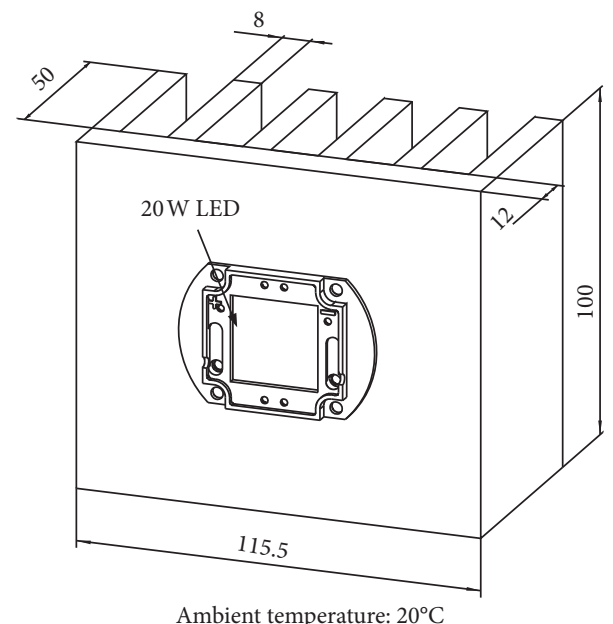

(b)

Figure 9: (a) Cylindrical heat sink and (b) rectangular plate-fin heat sink. 


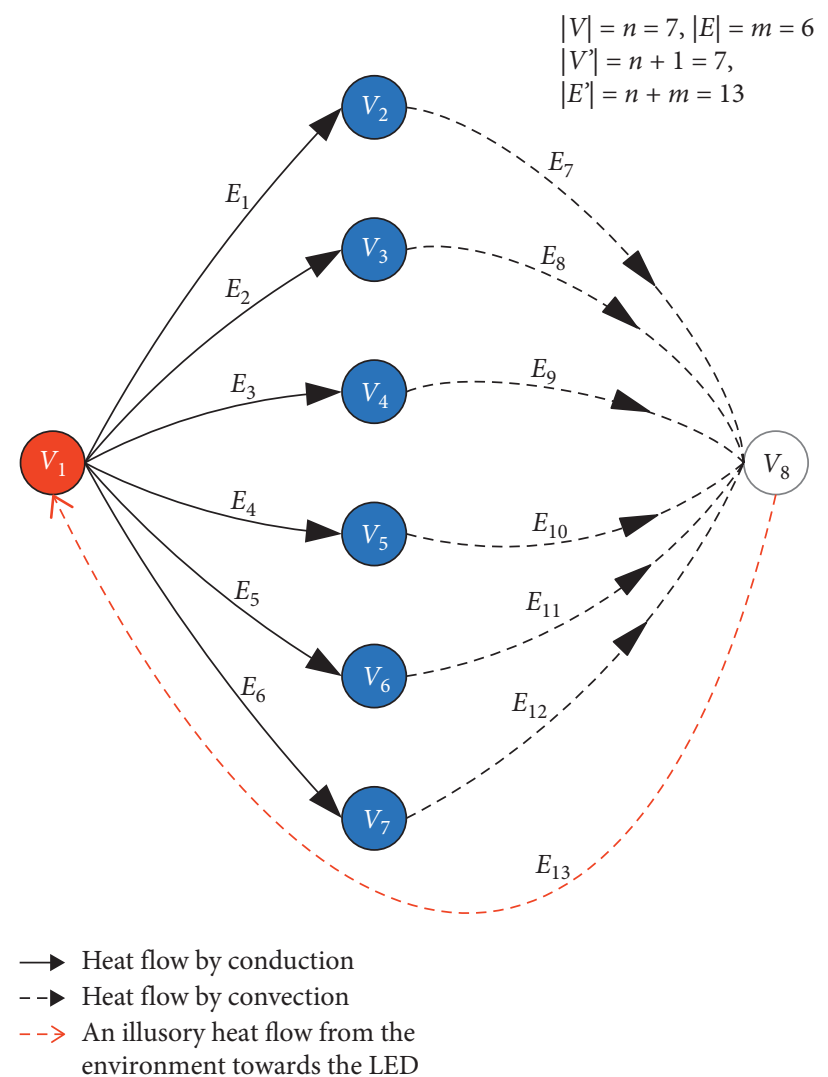

Figure 10: The digraph of the two heat sinks.

TABLe 3: The matrix representation of the heat flow graph.

The adjacency matrix $A_{H}$ by $n \times n(7 \times 7) \quad \begin{array}{lllllllll}\text { The specific matrix } \\ \text { Thix }\end{array}$

The incidence matrix $M_{F}$ by $(n+1) \times(m+n)(8 \times 13) \quad\left(\begin{array}{ccccccccccccc}1 & 1 & 1 & 1 & 1 & 1 & 0 & 0 & 0 & 0 & 0 & 0 & -1 \\ -1 & 0 & 0 & 0 & 0 & 0 & 1 & 0 & 0 & 0 & 0 & 0 & 0 \\ 0 & -1 & 0 & 0 & 0 & 0 & 0 & 1 & 0 & 0 & 0 & 0 & 0 \\ 0 & 0 & -1 & 0 & 0 & 0 & 0 & 0 & 1 & 0 & 0 & 0 & 0 \\ 0 & 0 & 0 & -1 & 0 & 0 & 0 & 0 & 0 & 1 & 0 & 0 & 0 \\ 0 & 0 & 0 & 0 & -1 & 0 & 0 & 0 & 0 & 0 & 1 & 0 & 0 \\ 0 & 0 & 0 & 0 & 0 & -1 & 0 & 0 & 0 & 0 & 0 & 1 & 0 \\ 0 & 0 & 0 & 0 & 0 & 0 & -1 & -1 & -1 & -1 & -1 & -1 & 1\end{array}\right)$

The flow matrix $C_{F}$ by $(n-1) \times(m+n)(6 \times 13)$ 
TABLE 4: Experimental temperature excesses of a $20^{\circ} \mathrm{W}$ LED chip mounted on a cylindrical heat sink and a rectangular plate-fin heat sink against calculation values at $20^{\circ} \mathrm{C}$ ambient temperature.

\begin{tabular}{|c|c|c|c|c|c|}
\hline $\begin{array}{l}\text { Ambient } \\
\text { relative } \\
\text { humidity (\%) }\end{array}$ & $\begin{array}{l}\text { Calculation } \\
\left({ }^{\circ} \mathrm{C}\right)\end{array}$ & $\begin{array}{c}\text { Experimental } \\
\text { temperature excesses on } \\
\text { a rectangular heat sink } \\
\left({ }^{\circ} \mathrm{C}\right)\end{array}$ & $\begin{array}{l}\text { Relative errors between } \\
\text { experiment and } \\
\text { calculation for the } \\
\text { rectangular heat sink (\%) }\end{array}$ & $\begin{array}{c}\text { Experimental } \\
\text { temperature excesses on } \\
\text { a cylindrical heat sink } \\
\left({ }^{\circ} \mathrm{C}\right)\end{array}$ & $\begin{array}{l}\text { Relative errors between } \\
\text { experiment and } \\
\text { calculation for the } \\
\text { cylindrical heat sink (\%) }\end{array}$ \\
\hline 50 & 22.8 & 24.10 & 5.39 & 22.60 & 0.88 \\
\hline 55 & 23.1 & 24.23 & 4.66 & 22.71 & 1.72 \\
\hline 60 & 23.3 & 24.49 & 4.86 & 22.95 & 1.53 \\
\hline 65 & 23.5 & 24.87 & 5.50 & 23.30 & 0.87 \\
\hline 70 & 24.1 & 25.35 & 4.95 & 23.76 & 1.43 \\
\hline 75 & 24.8 & 25.97 & 4.49 & 24.35 & 1.85 \\
\hline 80 & 25.4 & 26.70 & 4.86 & 25.05 & 1.42 \\
\hline 85 & 26.3 & 27.56 & 4.58 & 25.85 & 1.76 \\
\hline 90 & 27.2 & 28.54 & 4.68 & 26.75 & 1.67 \\
\hline 95 & 28.3 & 29.62 & 4.47 & 27.76 & 1.94 \\
\hline 100 & 29.5 & 30.37 & 2.86 & 28.88 & 2.14 \\
\hline
\end{tabular}

$$
\begin{aligned}
& C_{1}=\left\{e_{1}, e_{7}, e_{13}\right\}, \\
& C_{2}=\left\{e_{2}, e_{8}, e_{13}\right\}, \\
& C_{3}=\left\{e_{3}, e_{9}, e_{13}\right\}, \\
& C_{4}=\left\{e_{4}, e_{10}, e_{13}\right\}, \\
& C_{5}=\left\{e_{5}, e_{11}, e_{13}\right\}, \\
& C_{6}=\left\{e_{6}, e_{12}, e_{13}\right\} .
\end{aligned}
$$

Table 4 shows the experimental and calculated temperature excesses between the environment and the junction of $20^{\circ} \mathrm{W}$ LED mounted on a cylindrical heat sink and rectangular plate-fin heat sink in the humidity range of $50 \%-100 \%$. It suggests that the junction temperature of LED increases with the increase of humidity, which is consistent with the conclusion in Section 3.1.3 and the conclusions of other papers [10]. Besides, the two heat sinks with identical digraph and thermal admittance matrix performed closely, thus verifying the correctness of heat sinks with an identical heat digraph but different types having close cooling performance.

\section{Conclusion}

This study developed a mathematical model based on graph theory, in association with computer technology, in order to lay a foundation to evaluate the optimal design among a large number of heat sinks' fin arrangement swiftly. After analyzing the heat and mass transfer process of a single fin under moisture environment, the whole heat dissipation process transferred from LED chips into the air through a heat sink can be represented by a digraph and described by two criteria considering the conservation of energy. On the basis of previous work, the concept of improved thermal admittance considering the effect of humidity is proposed; therefore, the temperature-heat calculation equations of the whole heat sink are deduced.

Based on experimental results, the following statements are concluded: the junction temperature of $20 \mathrm{~W}$, $25 \mathrm{~W}, 30 \mathrm{~W}, 35 \mathrm{~W}$, and $40 \mathrm{~W}$ LED chips mounted on the same heat sink was measured at $50 \%-100 \%$ relative humidity to compare with the calculation results, and the relative errors between the calculated value and the experimental data are all within $5 \%$, thus verifying the calculation model we constructed under moisture environment.

The experimental and calculated temperature excesses between the environment and the junction of $20^{\circ} \mathrm{W}$ LED mounted on a cylindrical heat sink and rectangular plate-fin heat sink are in the humidity range of $50 \%-100 \%$, thus verifying the correctness of heat sinks with an identical heat digraph but different types having close cooling performance.

\section{Abbreviations}

$T_{\text {ambient }}$ : Ambient temperature $\left({ }^{\circ} \mathrm{C}\right)$

$h$ : $\quad$ Surface heat transfer coefficient $\left(\mathrm{W} \cdot \mathrm{m}^{-2} \mathrm{~K}^{-1}\right)$

Nu: $\quad$ Nussle number

Pr: $\quad$ Prandtl number

l: $\quad$ Characteristic length $(\mathrm{m})$

$v$ : $\quad$ Kinematic viscosity $\left(\mathrm{m}^{2} \cdot \mathrm{s}{ }^{-1}\right)$

$k$ : Thermal conductivity $\left(\mathrm{W} \cdot \mathrm{m}^{-1} \mathrm{~K}^{-1}\right)$

$g: \quad$ Acceleration of gravity $\left(\mathrm{m} \cdot \mathrm{s}^{-2}\right)$

$q$ : $\quad$ Thermal power (W)

$\theta$ : Temperature excess between the fin and the surrounding environment $\left({ }^{\circ} \mathrm{C}\right)$

$R: \quad$ Thermal resistance $\left(\mathrm{K} \cdot \mathrm{W}^{-1}\right)$

$L: \quad$ Length of the fin $(\mathrm{m})$

$\omega: \quad$ Relative humidity (\%)

$H: \quad$ Height of the fin (m)

$\delta: \quad$ The thickness of the fin (m)

Gr: $\quad$ Grashof number

$\beta$ : $\quad$ Volume expansion coefficient $(1 / \mathrm{K})$

Le: $\quad$ Lewis number

$\alpha: \quad$ Thermal diffusivity $\left(\mathrm{m}^{2} \cdot \mathrm{s}^{-1}\right)$

$I_{f g}: \quad$ Latent heat of moisture $\left(\mathrm{J} \cdot \mathrm{kg}^{-1}\right)$

$c_{p}: \quad$ Specific heat of moisture $\left(\mathrm{J} \cdot \mathrm{kg}^{-1} \mathrm{~K}^{-1}\right)$

$h_{d}: \quad$ Mass transfer coefficient $\left(\mathrm{kg} \cdot \mathrm{m}^{-2} \cdot \mathrm{s}^{-1}\right)$.

\section{Data Availability}

The data used to support the findings of this study are included within the article. 


\section{Conflicts of Interest}

The authors declare there are no conflicts of interest regarding the publication of this article.

\section{Acknowledgments}

This research was supported by the Research Program of Science and Technology at Universities of Inner Mongolia Autonomous Region (NJZY20241).

\section{References}

[1] M.-H. Chang, D. Das, P. V. Varde, and M. Pecht, "Light emitting diodes reliability review," Microelectronics Reliability, vol. 52, no. 5, pp. 762-782, 2012.

[2] B. Sun, X. Jiang, K.-C. Yung, J. Fan, and M. G. Pecht, "A review of prognostic techniques for high-power white LEDs," IEEE Transactions on Power Electronics, vol. 32, no. 8, pp. 6338-6362, 2017.

[3] X. Qian, J. Zou, M. Shi et al., "Development of optical-thermal coupled model for phosphor-converted LEDs," Frontiers of Optoelectronics, vol. 12, no. 3, pp. 249-267, 2019.

[4] L. Sun, J. Zhu, and H. Wong, "Simulation and evaluation of the peak temperature in LED light bulb heatsink," Microelectronics Reliability, vol. 61, pp. 140-144, 2016.

[5] J. X. Zhu and L. X. Sun, "Mathematical model and computation of heat distribution for LED heat sink," European Physical Journal Plus, vol. 131, no. 5, p. 179, 2016.

[6] D. Jang, S.-J. Park, S.-J. Yook, and K.-S. Lee, “The orientation effect for cylindrical heat sinks with application to LED light bulbs," International Journal of Heat and Mass Transfer, vol. 71, pp. 496-502, 2014.

[7] D. Jang, D. R. Kim, and K.-S. Lee, "Correlation of cross-cut cylindrical heat sink to improve the orientation effect of LED light bulbs," International Journal of Heat and Mass Transfer, vol. 84, pp. 821-826, 2015.

[8] D. Jang, S.-J. Yook, and K.-S. Lee, "Optimum design of a radial heat sink with a fin-height profile for high-power LED lighting applications," Applied Energy, vol. 116, no. 3, pp. 260-268, 2014.

[9] B. Lv and F. Xiong, "Mathematical calculation model for temperature distribution of LED lamp heat sinks," Bandaoti Guangdian/Semiconductor Optoelectronics, vol. 39, no. 2, pp. 229-233, 2018.

[10] B.-X. Lyu, Y.-R. Chen, and F. Xiong, "Mathematical calculation model and its verification for temperature distribution of LED lighting's heatsinks for plant growth in the summer greenhouse," Chinese Journal of Luminescence, vol. 39, no. 8, pp. 1115-1122, 2018.

[11] W. A. Khan, J. R. Culham, and M. M. Yovanovich, "Optimization of pin-fin heat sinks using entropy generation minimization," IEEE Transactions on Components and Packaging Technologies, vol. 28, no. 2, pp. 247-254, 2005.

[12] H. E. Ahmed, "Optimization of thermal design of ribbed flatplate fin heat sink," Applied Thermal Engineering, vol. 102, pp. 1422-1432, 2016.

[13] Y. Lv and S. Liu, "Topology optimization and heat dissipation performance analysis of a micro-channel heat sink," Meccanica, vol. 53, no. 15, pp. 3693-3708, 2018.

[14] K. Lampio and R. Karvinen, "Optimization of convectively cooled heat sinks," Microelectronics Reliability, vol. 79, pp. 473-479, 2017.
[15] A. Bar-Cohen, R. Bahadur, and M. Iyengar, "Least-energy optimization of air-cooled heat sinks for sustainability-theory, geometry and material selection," Energy, vol. 31, no. 5, pp. 579-619, 2006.

[16] G. Schmid, L.-H. Yang, T.-H. Yang, and S.-L. Chen, "Influence of inter-fin base length on thermal performance of free hanging horizontal base heat sinks," Applied Thermal Engineering, vol. 108, pp. 1226-1236, 2016.

[17] S.-H. Yu, K.-S. Lee, and S.-J. Yook, "Optimum design of a radial heat sink under natural convection," International Journal of Heat and Mass Transfer, vol. 54, no. 11-12, pp. 2499-2505, 2011.

[18] W. J. Min, S. W. Jeon, and Y. Kim, "Optimal thermal design of a horizontal fin heat sink with a modified-opening model mounted on an LED module," Applied Thermal Engineering, vol. 91, pp. 105-115, 2015.

[19] F. Harary, Graph Theory, Addison-Wesley Publishing Co, Boston, MA. USA, 2019.

[20] Y. Lu, Y. Wang, Y. Lu, and N. Ye, "Derivation of contracted graphs with ternary/quaternary links for type synthesis of parallel mechanisms by characteristic strings," Robotica, vol. 33, no. 3, pp. 548-562, 2015.

[21] A. D. Kraus and A. Bar-Cohen, Design and Analysis of Heat Sinks, Wiley, New York, NY, USA, 1995.

[22] M. H. Sharqawy and S. M. Zubair, "Efficiency and optimization of a straight rectangular fin with combined heat and mass transfer," Heat Transfer Engineering, vol. 29, no. 12, pp. 1018-1026, 2008. 